\title{
Joint replacement, dental surgery, and antibiotic prophylaxis
}

\author{
Andrew Grant, Ceri Hoddinott
}

Department of

Orthopaedic Surgery,

Cardiff Royal Infirmary, Cardiff

Andrew Grant, registrar

Ceri Hoddinott, senior

registrar

Correspondence to:

Mr Andrew Grant, Prince of

Wales Hospital, Rhydlafer, South Glamorgan.

BMF 1992;304:959

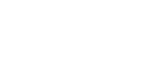

Numerous case reports support the assertion that secondary infection around prosthetic joints can occur after dental disease or manipulation, or both, but the need for prophylactic antibiotics remains controversial. In the United States prophylaxis is recommended, whereas in Britain individuals adopt their own policy. We surveyed orthopaedic surgeons and dental and general practitioners to determine their attitude towards prophylaxis in patients with prosthetic joints undergoing dental treatment.

\section{Subjects, methods, and results}

We sent a postal questionnaire to 125 each of orthopaedic surgeons, dentists, and general practitioners. Orthopaedic surgeons were asked whether any of their arthroplasty patients had had infections secondary to dental disease, about preoperative dental assessment, and whether they recommended prophylactic antibiotics for subsequent dental treatment. The dentists were asked about the need for prophylaxis in patients with prosthetic joints and those with cardiac lesions. General practitioners were asked about their prescribing habits in patients with prosthetic joints who had dental disease and about advice received from local orthopaedic surgeons. The table shows the response rates.

Response rate to survey about antibiotic prophylaxis for patients with prosthetic joints undergoing dental treatment

\begin{tabular}{lcc}
\hline & No sent surveys & No (\%) who returned surveys \\
\hline Orthopaedic surgeons & 125 & $104(83)$ \\
General practitioners & 125 & $98(78)$ \\
Dental practitioners & 125 & $101(81)$ \\
\hline
\end{tabular}

Ten of 104 orthopaedic surgeons reported infections thought to be secondary to dental disease, and two had isolated appropriate bacteria from infected joints. Four requested preoperative dental assessments, and 54 advised patients to request antibiotic prophylaxis during subsequent dental treatment. Only 33 gave similar advice to the general practitioner.

Only four of the 98 general practitioners were advised to give antibiotic prophylaxis to patients with prosthetic joints. Eighteen gave antibiotics to patients with subsequent dental infection.

Ninety one of the 101 dental surgeons gave prophy- laxis to patients with cardiac lesions but only 25 gave it to those with prosthetic joints. Dentists agreed that in appropriate patients prophylaxis was needed for all procedures causing gingival trauma and bleeding.

\section{Comment}

A causal relation between transient bacteraemia of dental origin and infection about a prosthetic joint has not been proved,' although a growing number of reports implicate dental disease in late sepsis. ${ }^{2} \mathrm{~A}$ report showing a temporal relation between dental treatment and infection and in which peptostreptococcus was isolated from the joint is particularly pertinent. ${ }^{3}$

In our survey $10 \%$ of participating surgeons believed that one or more of their patients had had late sepsis of dental origin. This is a surprisingly large report of sepsis from a potentially treatable source.

In a survey of American orthopaedic surgeons $93 \%$ (1488 of 1600) recommended antibiotic prophylaxis. ${ }^{1}$ Only $52 \%$ of our surgeons recommended that their patients ask for antibiotic prophylaxis for dental treatment and $32 \%$ advised general practitioners that these patients should receive prophylaxis. Only four (3\%) general practitioners reported receiving such advice.

There is wide agreement that antibiotic prophylaxis for dental treatment is necessary in high risk groups of patients with prosthetic joints. ${ }^{4}$ These groups include patients with diabetes mellitus, rheumatoid disease, and revised joints, and those who are immunosuppressed or taking steroids. Issuing patients with a joint replacement card may encourage dentists and general practitioners to prescribe prophylaxis.

The choice of antibiotic is important. Review of the bacteriology of infected prosthetic joints supports the use of broad spectrum drugs such as cephalosporins or penicillins with $\beta$ lactamase resistance. ${ }^{5}$

We recommend a preoperative dental check up before arthroplasty and that antibiotic prophylaxis should be given to patients receiving dental treatment causing gingival trauma or significant bacteraemia. Greater effort should be made by orthopaedic surgeons to inform general and dental practitioners of the need for antibiotic prophylaxis in patients with prosthetic joints receiving dental treatment.

1 Nelson JP, Fitzgerald RH, Jaspers MT, Little JW. Prophylactic antimicrobial coverage in arthroplasty patients. I Bone foint Surg [Am] 1990;72:1.

2 Linqvist C, Slatis P. Dental bacteraemia-a neglected cause of arthroplasty inqvist C, Slatis P. Dental bacteraemia -a
infections. Acta Orthop Scand 1985;56:506-8.

3 Sullivan PM, Johnston RC, Kelley SS, Des Moins DM. Late infection after total hip replacement caused by an oral organism after dental treatment. total hip replacement caused by an orat

4 Thyne G, Ferguson JW. Antibiotic prophylaxis during dental treatment in patients with prosthetic joints. F Bone foint Surg $[B r] 1991 ; 73: 191-4$.

5 Jacobson JJ, Matthews LS. Bacteria isolated from late prosthetic join infections: dental treatment and chemoprophylaxis. Oral Surg, Oral Med, Oral Pathol 1987;63:122-6.

(Accepted 4 February 1992)

\section{Antitoxins and botulinum toxin treatment}

Peter Hambleton, Helen E Cohen, Bryan J Palmer, Jack Melling

Injecting Clostridium botulinum type A toxin is now an established treatment for focal dystonia, ${ }^{12}$ but it is not a cure. Motor endplate regeneration generally leads to the re-establishment of symptoms; treatment is usually repeated at intervals of two to three months. Most patients respond well to chronic treatment, and there is good evidence that efficacy is maintained, ${ }^{3}$ but a minority of these patients stop responding ${ }^{34}$ for reasons that are not entirely clear. Antibody formation may be the cause in some patients. ${ }^{5}$ We report the results of a survey of serum samples from patients with torticollis who had had extended courses of treatment with toxin. Generally, such patients receive the highest doses of toxin and might be considered to be most likely to develop toxin neutralising antibodies.

\section{Patients, methods, and results}

From several hundred patients treated for spasmodic torticollis for some years with botulinum type A toxin, 10 who had maintained their response to treatment and 10 whose response had diminished appreciably were 